\title{
Transmission Behavior of Single-Mode Fiber Based on a Microchannel
}

\author{
M. NAdAfan ${ }^{a, *}$, A. Ahmadian ${ }^{b}$, \\ S.N. $\operatorname{ARAB}^{a}$ AND J.Z. ANVARI ${ }^{a}$ \\ ${ }^{a}$ Department of Physics, Faculty of Science, Shahid Rajaee Teacher Training University, \\ Tehran, P.O. Box 16788-15811, I.R. Iran \\ ${ }^{b}$ Department of Physics, Faculty of Science, Tarbiat Modares University, \\ Tehran, P.O. Box 14115-175, I.R. Iran
}

Received: 06.07.2020 \& Accepted: 12.02.2021

The properties of light transmission in a single-mode fiber (SMF) with a cylindrical microchannel were investigated by the FDTD method. Different microchannel diameters $(2-15 \mu \mathrm{m})$ were addressed. The core was made of silica with a refractive index of 1.4 which was covered by a carbon nanosheet whose refractive index was 2.47. The influence of the Fabry-Perot (FP) resonance was vividly observed in transmission properties. The transmission of light at a constant wavelength varied depending on the refractive index in the microchannel. The transmitted light decreased by increasing the microchannel refractive index. The transmission significantly changed with varying the microchannel refractive index from 1.3 to 1.4 and from 1.1 to 1.2. It was found that an SMF based on the microchannel at different diameters can be used for refractive index sensing. The numerical results are in good agreement with those reported in the microhole or microchannel experiment. The comparison of the related experimental and numerical results shows that the well-controlled microchannel diameter could increase light transmission by passing through the core-microchannel. The refractive index sensitivity to microchannel diameters is a promising approach for developing applications of fiber optical devices.

topics: optical fiber, single-mode fibers, transmission, index sensing

\section{Introduction}

Since the emergence of optical fiber technology in the 1960s, various applications of optical fiber have been developed [1]. The lasers also improve the optical fiber sensing devices in terms of sensitivity and accuracy [2]. Recently, femtosecond lasers have been extensively used in microchannel research in single-mode fibers (SMF) [3]. The ease of microchannel fabrication and restructuration in SMF have attracted great attention in terms of refractive index changes [3]. Due to the symmetry of the cylindrical shapes, the cylindrical microchannel is more interesting, especially for the refractive index sensing devices $[4,5]$. The refractive index properties are strongly dependent on the physical structure of the microchannel [4].

Utilizing different materials for core-cladding of optical fiber can enhance the refractive index sensing and accurate responses of fiber-optics devices. Moreover, the incorporation of dopants in fibers can alter the refractive index and hence influence the mortality rate in different wavelength ranges [3].
Numerical modeling can help to optimize the fiber structures using Maxwell's equations. The time-domain finite-difference (FDTD) method is a convenient way for simulating an SMF based on a microchannel [6-8].

Each fiber includes three parts: core, cladding, and surface. The core usually comprises a reflective material (for example, glass) set in the center of the fiber [4]. Since the development of the existing fibers and employing high strength fibers are necessary to maximize the utilization of fiber technology especially in the environments with disasters, introducing new fibers which are made of new materials can be interesting [9]. In this respect, here, the carbon nanosheet $(n=2.47)$ substituted glass. The cladding is composed of a material with a refractive index of 2.473. Given that several methods have been proposed to achieve the desired refractive index, it is assumed that this type of a material exists [9].

The properties of light transmission in an SMF based on a cylindrical microchannel are investigated in this research. For this purpose, a continuous 
wave was used to analyze the electric field caused by the change of the microchannel refractive index from 1 to 1.4. The electric field resulting from light transmission properties in carbon nanosheetcoated fiber with a cross-section microchannel was also explored. The transmittance spectra of the microchannel by different diameters were evaluated as well. Our theoretical data is in good agreement with experimental results. The effect of different core materials on the transmission is a new subject that has not received much attention during the past years. However, it can be a helpful parameter in the optical fiber application and the transmission behavior of the optical fiber.

\section{Optical fiber simulation: theory and modeling}

An optical fiber is indeed a cylindrical waveguide composed of two concentric cylinders with different diameters: the core, with a larger refractive index $\left(n_{1}\right)$, and the cladding, with a smaller refractive index $\left(n_{2}\right)$. The core and cladding are made of dielectric materials. The simplest form of an optical fiber includes a glass core coated by a layer with a lower refractive index. Therefore, the light beam directed toward the end of the fiber is limited by the overall reflection within the cores. A cylindrical microchannel intersecting the fiber core attracts more attention in the research field, especially in the application involving refractive index-related sensing.

Regarding the direction of wave propagation and the distribution of the lateral electric field in the light propagation direction, a $200 \mu \mathrm{m}$ cylinder was considered. Moreover, the core and cladding diameters of the fiber were set as $8 \mu \mathrm{m}$ and $60 \mu \mathrm{m}$, respectively. The CNS fiber cladding with a refractive index of 2.47 coated the core whose refractive index was by 0.003 greater $(2.473)$.

It should be noted that the results were obtained for five different microchannel diameters including $2,4,5,8$ and $15 \mu \mathrm{m}$. The microchannel was perpendicular to the light propagation direction.

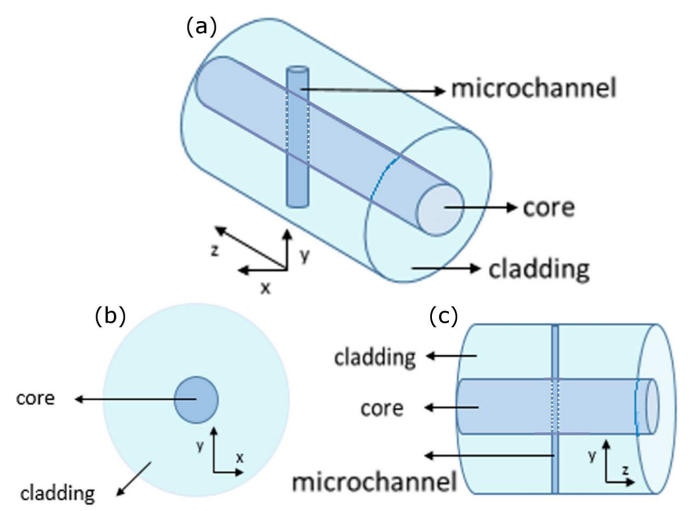

Fig. 1. Schematic diagram of a microchannel passing through the core of an SMF.
To observe the microchannel-guided wave propagation and the distribution of field along the propagation direction, a fiber length must be at least equal to $200 \mu \mathrm{m}$. Depending on the SMF on the fiber, the computation area can be confined to a rectangle $(40 \mu \mathrm{m} \times 40 \mu \mathrm{m})$ in the middle of which the core was set. The cross-section of such a system is shown in the inset of Fig. 1. It should be stressed that the scattering of the guided wave by a microchannel is a $3 \mathrm{D}$ problem but it was simplified to a $2 \mathrm{D}$ one. This simplification will not reduce the accuracy of our calculation by the FDTD algorithm. In this simulation, the microchannel operates like a scatter in the core and a Fabry-Perot (FP) interferometer made of two concave mirrors. Both the scattering and resonating of the FP cavity are illustrated in the inset of Fig. 1b. The refractive index of the core and its surroundings determined the reflectivity.

\section{Simulation result and discussion}

In this study (a 2D scattering problem), the TE polarization $\left(H_{x}, E_{y}, H_{z}\right)$ is assumed by the $E_{y}$ direction along the microchannel. The electric and magnetic fields components are related to each other in the following way:

$$
E^{(n+1)}=E^{(n)}+\frac{\Delta t}{\varepsilon}\left(\nabla \times H^{\left(n+\frac{1}{2}\right)}\right)
$$

and

$$
H^{\left(n+\frac{3}{2}\right)}=H^{\left(n+\frac{1}{2}\right)}-\frac{\Delta t}{\mu}\left(\nabla \times E^{(n+1)}\right) .
$$

Therefore, in order to obtain the electric field component, the Fourier transform in terms of time must be taken as the following equation:

$$
E(\omega)=\int_{0}^{T} \mathrm{~d} t E(t) \mathrm{e}^{\mathrm{i} \omega t} .
$$

Thus, the electric field is obtained at any particular frequency or wavelength.

The lowest mode is considered because of simplicity in the calculation of its distribution. Furthermore, the CW excitation can be used for obtaining the transmission spectrum. Considering the longitudinal Poynting vector, $S=\frac{1}{2} \operatorname{Re}\left(E \times H^{*}\right)$ along the output plane, the power spectral density and transmission spectrum can be evaluated.

\subsection{Scattering electric field distribution}

For the sake of simplicity, the simulation area was converted from 3D to 2D. A CNS-coated fiber with a refractive index of 2.47 and a core with a refractive index of 2.473 was considered. The microchannel was perpendicular to the optical fiber.

The microchannel refractive indices change from 1.0 to 1.4. The transmission properties of light through a microchannel with different diameters were evaluated. The wave propagation was driven by a $4,5,8$ and $15 \mu$ m-diameter microchannel using a CW excited wavelength of the order of $1550 \mathrm{~nm}$. 


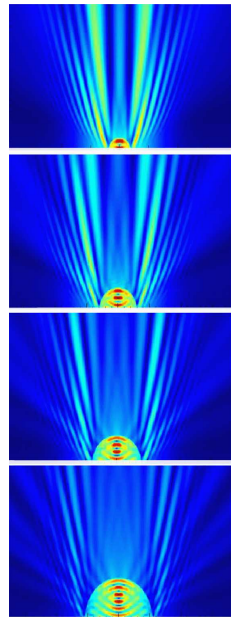

(a)
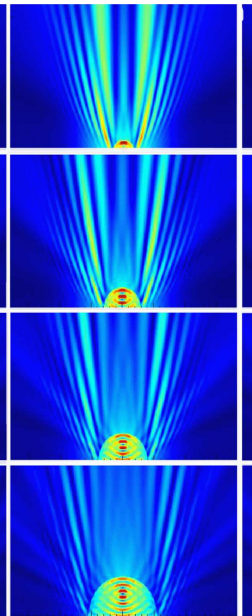

(b)
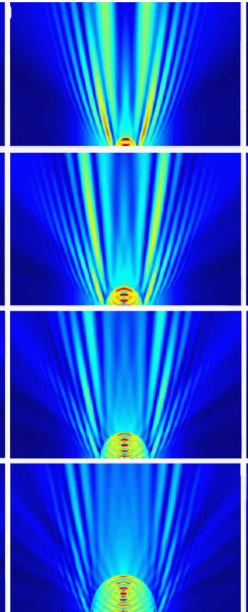

(c)

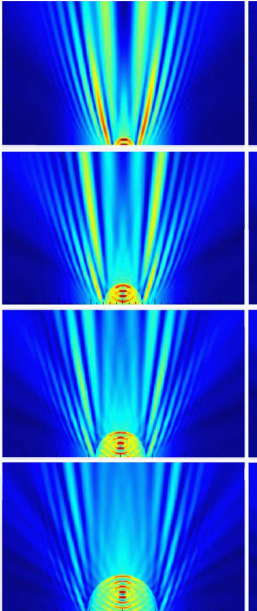

(d)
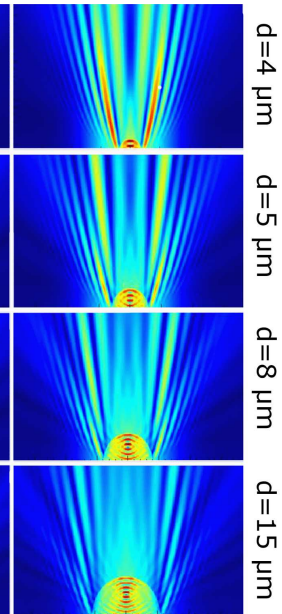

(e)

Fig. 2. The $E_{y}$ field distribution of the guided wave scattered by the microchannel with $4,5,8$ and $15 \mu \mathrm{m}$ diameter with different refractive indices of (a) $n=1$, (b) $n=1.1$, (c) $n=1.2$, (d) $n=1.3$, and (e) $n=1.4$.

The distribution of the electric field at each time step was measured for the microchannel with different refractive indices. The profiles of the electric field with different diameters and various refractive indices are presented in Fig. 2.

According to Fig. 2, the scattering and recoupling of the scattered light were strongly related to the microchannel refraction coefficient. It should be noted that the transmission of the fiber depended on the microchannel structure and the light wavelength. In fact, the light transmitted through the fiber has a linear response in terms of the refractive index of the microchannel.

Mostly, the light emitted by the waveguide is dispersed after being scattered in the fiber core of the microchannel. The light returned to the core provided conditions for the total internal reflection and phase matching at the core-cladding boundary which can be assigned to both roles of the FP cavity (scattering, reflecting). The variation of the refractive index in the microchannel not only varies the reflectance of the core-channel boundary but also alters the optical pathway between the two mirrors. The change in the optical pathway is directly related to the resonance wavelength of the FP cavity.

Similar changes were observed in the electric field distribution of microchannels with different diameters and refractive indices. It can be seen that by increasing the refractive index of the microchannels with different diameters, the electric field in the center decreased. Based on the results in Fig. 2, it can be concluded that the microchannel center has a higher electric field intensity when compared to the other regions. At the constant refractive index, an increment in the diameter of the microchannel will decline the electric field intensity. This result was confirmed in different refractive indices of the microchannel/microhole by other researchers $[6,10]$.

\subsection{Microchannel transmission properties}

The transmission properties of the SMF were evaluated using the microchannel crossing the fiber core. The refractive index of the microchannel was changed from 1.0 to 1.4. The light wavelength and its spectral width were $1550 \mathrm{~nm}$ and $40 \mathrm{~nm}$, respectively.

Figure 3 represents the transmission dependence on the microchannel refractive index for various wavelengths. The transmission vividly changed with the refractive index of the microchannel. The changes in the transmission were more observable in $4 \mu \mathrm{m}, 5 \mu \mathrm{m}, 8 \mu \mathrm{m}$ and $15 \mu \mathrm{m}$ diameter of the microchannel. Herein, the highest and lowest transmission belonged to the microchannels with the respective diameters of $15 \mu \mathrm{m}$ and $2 \mu \mathrm{m}$, regardless of the wavelength. However, the overall transmittance has the smallest value for the smallest microchannel.

In the case shown in Fig. 3, when increasing the refractive index of the microchannel, the recorded transmission decreased. Decreasing transmittance is independent of the wavelength of incident light. The maximum transmittance is related to the $15 \mu \mathrm{m}$ diameter of the microchannel in each refractive index of the microchannel. The obtained results are in line with the previous reports $[3,11]$. Therefore, the transmitted light decreased by increasing the refractive index of the microchannel.

The transmission curves in Fig. 3a-e show periodical fluctuation with the increase of the refractive index; although the fluctuation amplitude is reduced, the period decreases with the increase of the microchannel diameter, which is similar to the characteristics of the typical FP cavity.

Qui et al. [6] simulated the SMF in microchannels with diameters of $4,6,8$, and $11 \mu \mathrm{m}$ and the refractive index of the core material was 1.4. In the present study, the refractive index equals 2.47 . 


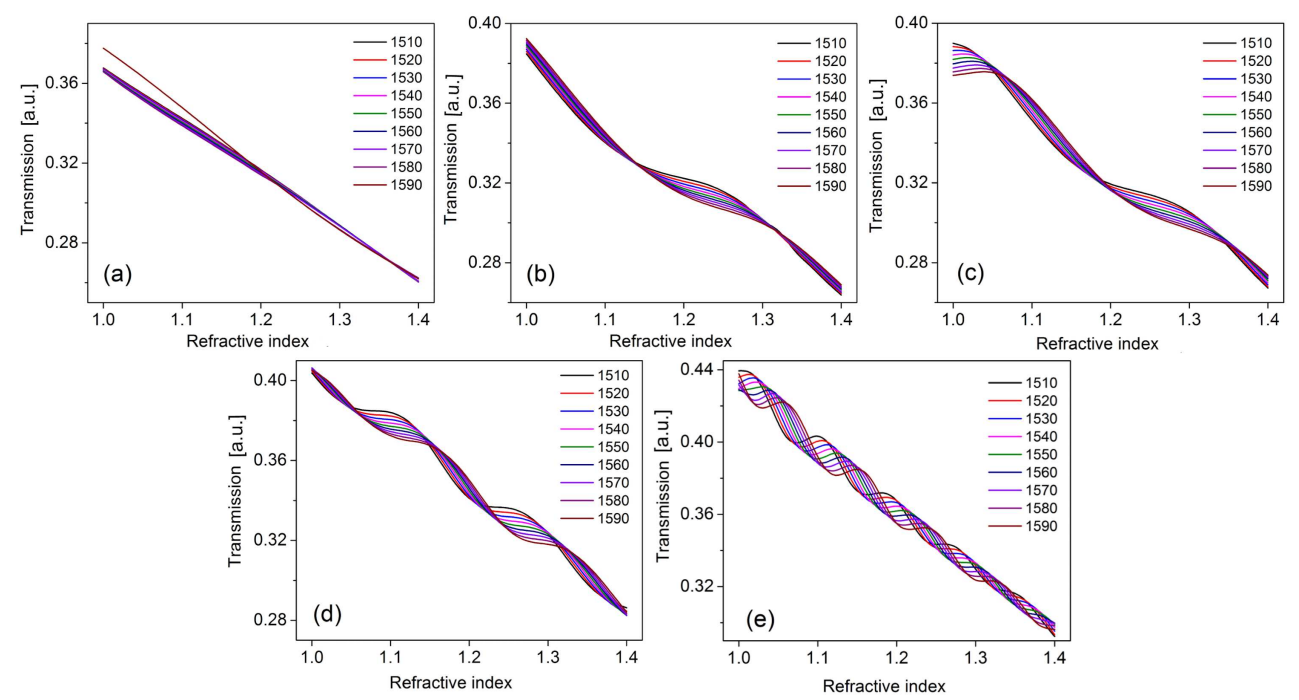

Fig. 3. Transmission light for different refractive indices of the microchannel in (a) $2 \mu \mathrm{m}$, (b) $4 \mu \mathrm{m}$, (c) $5 \mu \mathrm{m}$, (d) $8 \mu \mathrm{m}$, and (e) $15 \mu \mathrm{m}$ microchannel diameters.
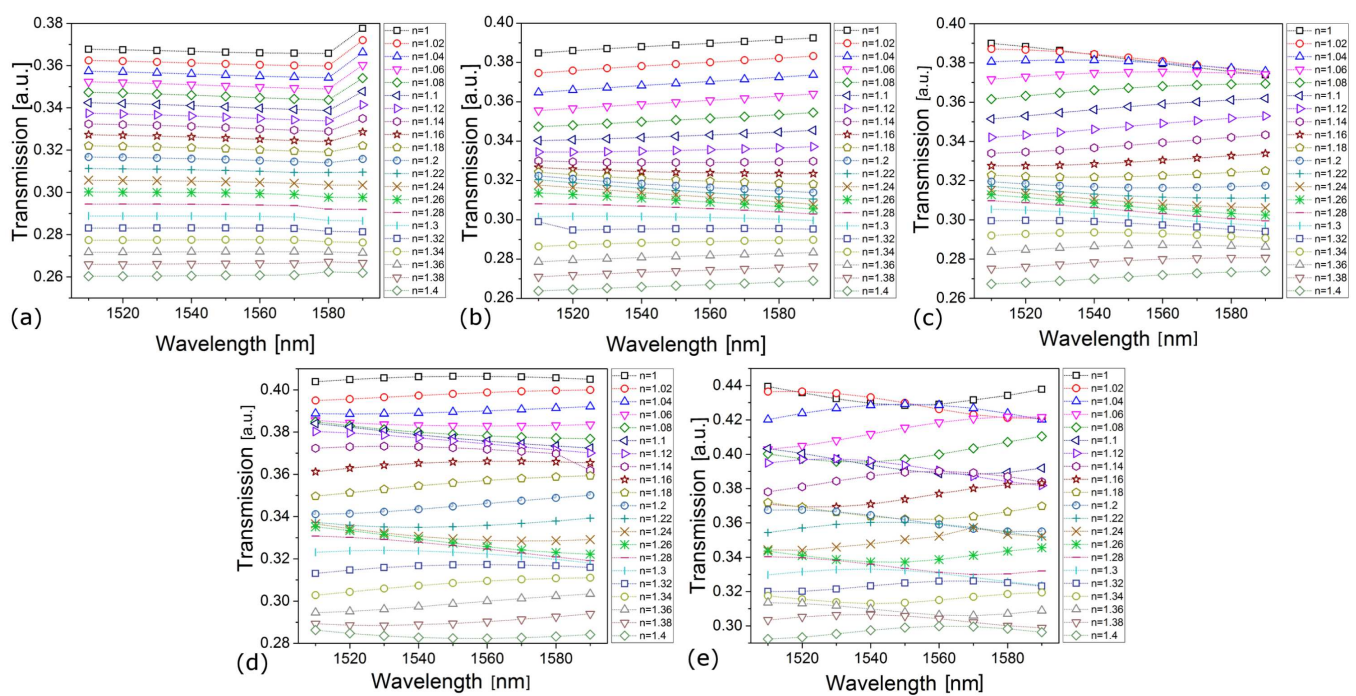

Fig. 4. Transmission light vs. different wavelengths for (a) $2 \mu \mathrm{m}$, (b) $4 \mu \mathrm{m}$, (c) $5 \mu \mathrm{m}$, (d) $8 \mu \mathrm{m}$, and (e) $15 \mu \mathrm{m}$ microchannel diameters.

Qui and his co-workers reported that the microchannel diameter in the range of $4-6 \mu \mathrm{m}$ is the most adequate size for optimizing the transmission. The transmission of all microchannel diameters considered by us showed similar behavior to those reported [6]. Recently, an SMF-based microhole has been fabricated and simulated [10] whose results are in agreement with our findings. Zhou et al. [10] used a microhole with a diameter of $6 \mu \mathrm{m}$ fabricated in an SMF by a femtosecond laser. The Zhou experiment showed that in a specific region of the refractive index, the transmission of a microhole increased. Interestingly, in our numerical calculations as well as in the experimental results, the nonlinear changes of transmission vs. the refractive index of the microchannel or microhole coincided with each other.
As it was mentioned before, the microchannel is similar to an FP cavity as it is dependent on the light wavelength. Changes in the transmission, if any, are usually small due to the low reflectivity of the core-microchannel boundary. However, the free spectral range of the FP cavity is larger than the wavelength variations and consequently different wavelengths lower than $1580 \mathrm{~nm}$ will exhibit similar behaviors by the refractive index changes with different diameters of the microchannel (see Fig. 4). If the core has a lower diameter than the microchannel, different wavelengths do not influence the transmission. Thus, in the low refractive index, by increasing the wavelength in $4 \mu \mathrm{m}$ diameter of the microchannel, the transmission increased. By increasing the diameter of the microchannel, the increasing wavelength increases 
the transmission rather than decreases it. However, at large microchannel diameters, there is a little irregularity in the transmission behavior. Also, in the microchannel diameters much smaller than the core diameter, the turning point of wavelengths is $1580 \mathrm{~nm}$. In the lower refractive index, the direction change of the transmission was much more visible than a higher refractive index. In the much larger diameter than the core diameter, the same behavior for the transmission versus the wavelength is not observed.

To make a comparison between these results and a similar experimental work, one should refer to a research concerning tilted fiber Bragg gratings (TFBG) and their refractive index sensory [12]. By detecting the transmission power of TFBGs, Miao and co-workers [12] measured the surrounding refractive index (SRI). Some SRI sensors have been proposed as chemical-biochemical sensors which in fact are based on TFBGs $[12,13]$. According to their report, when the refractive index is changing within the range of $1.37-1.42$, the transmission power decreases. One of the ways to improve the detected range of SRI and sensitivity of TFBG is to reduce the cladding diameter [14]. Our numerical data and transmission behavior of the simulated system in the present research agree with experimental data reported by Miao's research. Furthermore, Tao's research [15] referred to a similar idea regarding hollow silica nanomaterials and the refractive index engineering technology. Using such a technology, films consisting of shape-tunable hollow silica nanomaterials were prepared with refractive indices in the range of 1.045 to 1.426 . This kind of approach enables a new generation of film materials with a very low refractive index.

Another optical property very important when designing materials is the effective refractive index. Based on the FDTD method, the effective refractive index of the slab has been calculated and presented in Fig. 5.

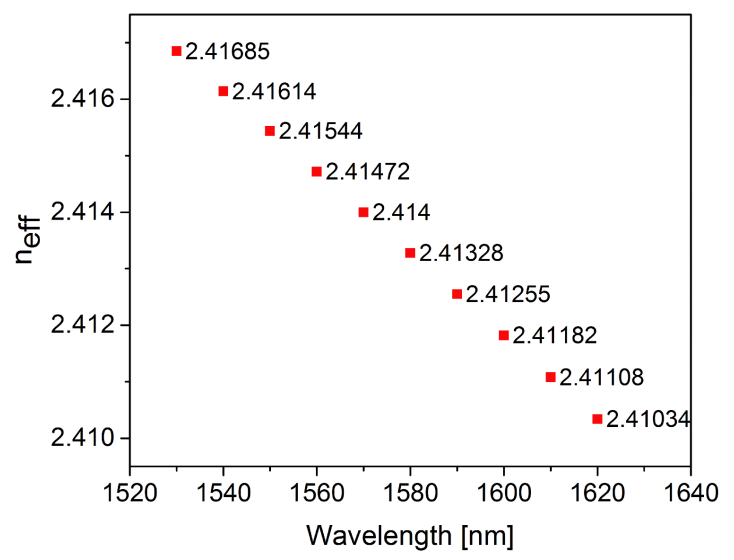

Fig. 5. The effective refractive index for the material $n=2.47$, for several wavelengths (1520$1620 \mathrm{~nm})$.
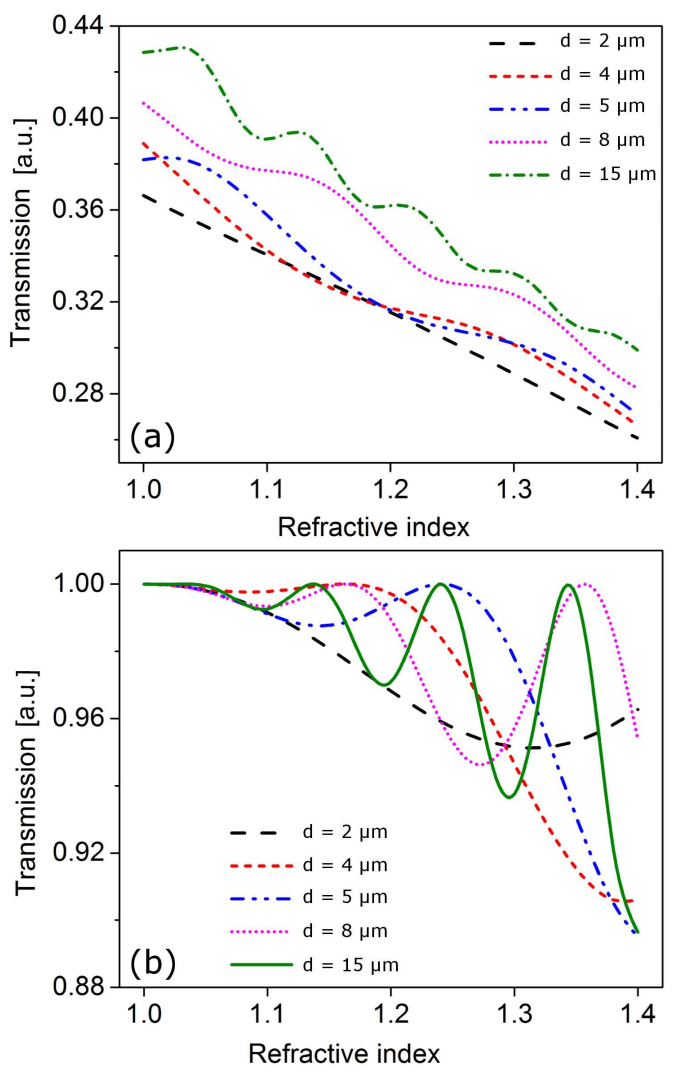

Fig. 6. (a) The calculated transmission of a crosssectional of microchannel different diameters, and (b) the transmission of a typical FP cavity with different cavity lengths $h=d / 2$, versus a different refractive index of the microchannel in $1550 \mathrm{~nm}$.

Figure 6a shows the transmission properties of microchannels with different diameters with a step size of 0.02 in $1550 \mathrm{~nm}$, in the refractive index range of 1.0-1.4. The periodic fluctuations could be observed by increasing the microchannel diameter. The period of fluctuations increased, whereas the amplitude of fluctuations decreased. Interestingly, the transmission of the FP cavity is the same as the transmittance of the above-simulated system [16] according to

$$
T_{t}=\frac{(1-R)^{2}}{(1-R)^{2}+4 R \sin ^{2}\left(\frac{2 \pi}{\lambda} n h\right)} .
$$

Here, $h, n$ and $R$ are the cavity length, the refractive index of the FP cavity and the reflectivity of the mirrors, respectively. Figure $6 \mathrm{~b}$ shows the transmittance of the FP cavity in which the length of the cavity $h$ is one half of the microchannel diameter $(h=d / 2)$. When the refractive index of the SMF microchannel is changed from 1.0 to 1.4 , the period of transmission fluctuations is similar to the transmission fluctuations of the FP cavity with the cavity length of $h=d / 2$. One might suspect that the scattering effect influences transmission fluctuations [6]. And indeed, the transmission characteristics of this system result as a combination of the scattering and FP resonance effects. 


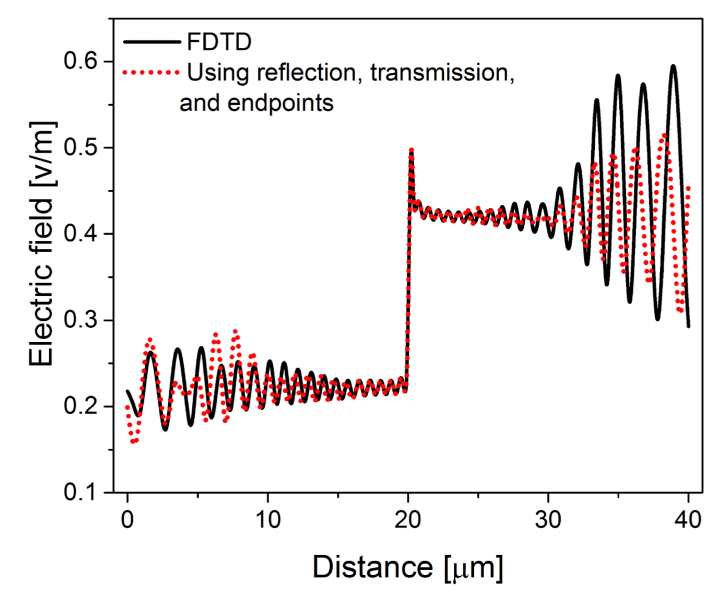

Fig. 7. The electric field behavior of the two methods into the fiber axis.

A similar experimental report recorded the transmission changes and the loss of transmission changes of the microchannel in the range of 1.2 to 1.4 refractive index for $4,6,8$ and $11 \mu \mathrm{m}$ microchannel diameter in $1550 \mathrm{~nm}$ wavelength [17]. The transmission changes of different microchannels in Fig. 6 are in good agreement with the experimental results reported in [17].

In order to find the accuracy of the simulated system, we examined our method with the use of another method. For simplicity, the source has been considered as an emitter of periodic plane waves and the electric fields have been studied in the propagation direction of the fiber. The sizes of the fiber and microchannel are the same size as in our system. The system is subjected to the electric fields of the source, which is located outside of the microchannel. The electric field of the system is investigated by the FDTD method. In another method, the electric field has been studied by employing the reflection, transmission, and endpoint phenomena. To this end, the microchannel is considered in an environment with a refractive index of 2.47 , while the source radiation axis is perpendicular to the microchannel axis.

Inside the microchannel, the electric field transmission from the fiber to the microchannel and reflection have been considered as the most effective aspects. Outside of it, however, the impact of microchannel endpoints (such as new sources) has received more attraction. Taking into account both phenomena, the electric field error ratio is achieved at the level of $8 \%$. It should be noted that in the case of the second method, a large approximation has been employed to simplify. The obtained results for electric field propagation in the direction of the fiber axis together with the comparison of both methods are presented in Fig. 7. In the simulation, the wavelength was $1590 \mathrm{~nm}$, the source has been located at $20 \mu \mathrm{m}$ and microchannel index was $n=1$.

\section{Conclusion}

In the present research, the simulation of a CNScoated SMF with a microchannel intersecting the core was addressed. The numerical results were obtained using the FDTD (2D) method. Different diameters of the microchannel (from $2 \mu \mathrm{m}$ to $15 \mu \mathrm{m}$ ) show different transmission properties in the refractive index range 1.0-1.4. The calculated transmission vs. refractive index of the microchannel shows good agreement with the reported experimental results, which confirms and validates this research. The numerical transmitted light decreased by incrementing the microchannel refractive index. For the core of SMF with a diameter of $15 \mu \mathrm{m}$, the microchannel exhibited the most changes in transmission - it can be therefore considered as the most suitable candidate for refractive index sensing purposes. The transmission results indicated the crucial role of the FP resonance effect in the transmission characteristics. The simple core-microchannel structure can be proposed for refractive index sensing applications due to its well-controllable diameter since the diameter size can remarkably influence the transmission rate of optical fibers.

\section{Acknowledgments}

The work was supported by the Shahid Rajaee Teacher Training University.

\section{References}

[1] G.P. Agrawal, P.L. Kelley, I.P. Kaminow, Nonlinear Fiber Optics, 3rd Ed., Academic Press, 2001.

[2] K. Barczak, Acta Phys. Pol. A 122, 793 (2012).

[3] R. Buczynski, Acta Phys. Pol. A 106, 141 (2004).

[4] T. Wolinski, A. Domanski, Acta Phys. Pol. A 103, 211 (2003).

[5] C. Mou, K. Zhou, E. Davies, L. Zhang, I. Bennion, Photon. Technol. Lett. 21, 1559 (2009).

[6] J. Qiu, W. Hong, D.N. Wang, D. Gao, Y. Wang, Y. Li, Opt. Fiber Technol. 17, 580 (2011).

[7] X. Miao, X. Yuan, H. Lv, H. Zhou, W. Zheng, G. Zhou, Optik 127, 168 (2016).

[8] N. Muduli, G. Palai, Optik 125, 7116 (2014).

[9] H.W. Ji, S.S. Yoo, J. Kim, D.D. Koo, Water 10, 983 (2018).

[10] J. Zhou, Y. Zheng, Optik 185, 1 (2019).

[11] J. Petrovic, Y. Lai, I. Bennion, Appl. Optik 47, 1410 (2008). 
[12] X. Shu, B.A.L. Gwandu, Y. Liu, L. Zhang, I. Bennion, Opt. Lett. 26, 774 (2001).

[13] L.Y. Shao, A.P. Zhang, W.S. Liu, H.Y. Fu, S. He, IEEE Photon. Technol. Lett. 19, 30 (2007).

[14] Y.P. Miao, B. Liu, Q.D. Zhao, Opt. Fiber Technol. 15, 233 (2009).
[15] C. Tao, X. Zou, K. Du, L. Zhang, H. Yan, X. Yuan, Opt. Lett. 43, 1802 (2018).

[16] C.M. Furse, O.P. Gandhi, IEEE Microw. Guided Wave Lett. 5, 326 (1995).

[17] Y. Wang, D.N. Wang, M. Yang, W. Hong, P. Lu, Opt. Lett. 34, 3328 (2009). 